\title{
Perfil dos pacientes internados em hospitais universitários de grande porte: conhecer para potencializar a assistência
}

\author{
Profile of inpatients in large universitary hospitals: know to enhance the \\ assistance
}

Pollyana Ruggio Tristão Borges, Débora Ebert Fontes, Danielle Latini Gaudêncio, Rosana Ferreira

\begin{abstract}
Como citar este artigo: BORGES, POLLYANA R. T.; FONTES, DÉBORA E.; GAUDÊNCIO, DANIELLE L.; SAMPAIO, ROSANA F.; Perfil dos pacientes internados em hospitais universitários de grande porte: conhecer para potencializar a assistência. Revista Saúde (Sta. Maria). 2020; 46 (2)
\end{abstract}

\section{Autor correspondente: Nome: Pollyana Ruggio Tristão Borges E-mail: pollyanaborges. fisio@gmail.com Telefone: (3 1) 998840407 Formação Profissional: Formada em Fisioterapia pela Universidade Federal de Minas Gerais (UFMG), Belo Horizonte, Minas Gerais, Brasil. Mestre em Ciências da Reabilitação pelo Programa de Pós- graduação em Ciências da Reabilitação da UFMC. Atualmente discente de doutorado do mesmo programa.}

Filiação Institucional: Universidade Federal de Minas Gerais

Endereço para correspondência:

Rua: Avenida Antônio

Carlos, 6627

Bairro: Pampulha

Cidade: Belo Horizonte Estado: Minas Gerais

CEP: $31270-90$

Data de Submissão: 20/04/2020

Data de aceite: 21/09/2020

Conflito de Interesse: Não há conflito de interesse \author{
Sampaio
}

RESUMO

Objetivo: Descrever o perfil sociodemográfico, clínico e funcional dos pacientes atendidos pela fisioterapia, internados nas enfermarias de hospitais universitários de Belo Horizonte. Métodos: Participaram 200 adultos, de ambos os sexos, internados nas enfermarias do Hospital das Clínicas da Universidade Federal de Minas Gerais e do Hospital Risoleta Tolentino Neves, em atendimento fisioterápico. As informações foram coletadas no prontuário e por meio de perguntas ao paciente/acompanhante. A funcionalidade nos domínios de mobilidade básica, atividade diária e cognição aplicada foram avaliadas pelo "6-clicks", instrumento desenvolvido com o arcabouço teórico da Classificação Internacional de Funcionalidade, Incapacidade e Saúde que avalia especificamente pacientes hospitalizados. Para análise, utilizou-se medidas de tendência central, dispersão e frequência, e testes comparativos não paramétricos. Resultados: A amostra foi composta por sujeitos com idade média de $59 \pm 17,13$ anos, com baixos níveis de escolaridade e renda, de forma que a maioria residia em outro município (52\%). Foi observado tempo prolongado de internação de 27,1 $\pm 29,93$ dias, presença de comorbidades, reinternações frequentes e predominância de condições neurológicas e cardíacas. A média de atendimentos fisioterápicos foi de 23 sessões e 63,5\% recebiam acompanhamento de nutricionistas. Em quase todos os setores dos hospitais, a mobilidade básica foi o domínio mais comprometido, seguido de atividade diária e cognição aplicada $(p<0,05)$. Os idosos apresentaram maiores déficits em atividade diária, bem como aqueles internados há pelo menos 30 dias $(p<0,05)$. Os pacientes mais jovens obtiveram melhores resultados no domínio cognição aplicada, enquanto aqueles com menor tempo de escolaridade apresentaram piores resultados $(p<0,05)$. Conclusão: Este estudo evidenciou informações sociodemográficas, clínicas e funcionais relevantes de pacientes internados em hospitais universitários de grande porte. Os dados obtidos ressaltam a importância da alocação de recursos, da criação de políticas públicas e de programas de prevenção e promoção da saúde com a finalidade de reduzir os impactos da hospitalização e potencializar a assistência.

PALAVRAS-CHAVE: Pacientes Internados; Fisioterapia; Atividades Cotidianas.

\section{ABSTRACT}

Objective: To describe the sociodemographic, clinical and functional profile of patients attended by physiotherapy and admitted to the medical wards of large university hospitals in Belo Horizonte. Methods: 200 adults, of both sexes, that were admitted to the wards of the Hospital das Clínicas of the Federal University of Minas Gerais and the Risoleta Tolentino Neves Hospital, and that were in physiotherapy treatment. Informations were collected from medical records and through questions to the patient/companion. Functioning in the domains of basic mobility, daily activity and applied cognition were assessed using "6-clicks", instrument developed with conceptual framework of the International Classification of Functioning, Disability and Health. For analysis, measures of central tendency, dispersion and frequency, and non-parametric comparative tests were used. Results: The sample was composed of subjects with mean age of $59 \pm 17.13$ years old, low income and education, and the most part from another city $(52 \%)$. Prolonged hospital stay of $27 \pm 29.93$ days, presence of comorbidities, frequent readmissions and predominance of neurological and cardiac conditions were observed. The average of physiotherapy attendance was 23 sessions, and $63.5 \%$ received assistance from nutritionists. In almost all sectors of hospitals, basic mobility was the most compromised domain, followed by daily activity and applied cognition $(p<0.05)$. The elderly presented more deficits in daily activity as well as those hospitalized for at least 30 days $(p<0.05)$. The youngest obtained best results in the domain of applied cognition while those with lower education presented worse results in this domain $(p<0.05)$. Conclusion: This study evidenced relevant sociodemographic, clinics and functional information of patients admitted to large university hospitals. The data obtained highlight the importance of allocating resources, creating public policies and prevention and promotion health programs with the aim of reduce negative impacts of hospitalization and enhance assistance.

KEYWORDS: Inpatients; Physiotherapy; Activities of Daily Living. 


\section{INTRODUÇÃO}

A hospitalização é um período desafiador para o indivíduo devido, entre outros fatores, às mudanças no ciclo circadiano, no hábito alimentar, na mobilidade e na ingesta de medicamentos com seus efeitos colaterais ${ }^{1}$. Pacientes hospitalizados, mais frequentemente os idosos, apresentam dificuldades para fazer transferências, comer, caminhar, vestir, realizar a higiene pessoal e se comunicar com os familiares ${ }^{2}$. A literatura discute que a mobilidade reduzida em idosos prediz declínio funcional nas atividades de vida diária ${ }^{3}$, reinternação ${ }^{4}$, institucionalização e morte 5 . Durante a internação, os pacientes de todas as idades passam mais da metade do tempo deitados no leito e pouco tempo é dedicado a pequenas caminhadas no hospital ${ }^{6}$. Um estudo mais recente mostrou que limitações em atividades de mobilidade, de autocuidado e também de cognição caracterizam uma redução da funcionalidade e podem interferir diretamente no quadro clínico, no tempo de hospitalização e na funcionalidade dos pacientes após a alta hospitalar?

Neste contexto, pacientes hospitalizados necessitam do cuidado de equipes de reabilitação para atender as suas necessidades e avaliar e intervir nas limitações apresentadas ${ }^{8}$. As ferramentas padronizadas que investigam a funcionalidade permitem conhecer as necessidades clínicas e funcionais dos pacientes e monitorar os resultados das intervenções ao longo do tempo. $O$ uso destas ferramentas auxilia a tomada de decisões, facilita a comunicação entre profissionais e setores do hospital, promove uma atenção integral ao indivíduo, e, ainda, subsidia a gestão financeira do serviço ${ }^{8}$. O conhecimento de informações sociodemográficas, clínicas e funcionais favorece o planejamento racional de recursos financeiros e humanos, contribui para um melhor encaminhamento do paciente entre profissionais e setores do hospital, além de auxiliar na elaboração de políticas públicas e estratégias de prevenção e promoção da saúde ${ }^{9,10}$. Por isso, uma investigação do perfil dos indivíduos hospitalizados é fundamental.

Em Belo Horizonte, o Hospital das Clínicas da Universidade Federal de Minas Gerais (HC-UFMG) e o Hospital Risoleta Tolentino Neves (HRTN), hospitais universitários de grande porte, oferecem atendimento para a população do município e do Estado de Minas Gerais pelo Sistema Único de Saúde (SUS) em todas as especialidades. Esses hospitais contam também com profissionais de reabilitação como fisioterapeutas, terapeutas ocupacionais, nutricionistas, fonoaudiólogos, dentre outros. Diante disso, o objetivo desse estudo foi descrever o perfil sociodemográfico, clínico e funcional dos pacientes internados nas enfermarias de dois hospitais universitários de grande porte da cidade de Belo Horizonte, atendidos pela fisioterapia.

\section{MÉTODO}

Trata-se de um estudo observacional do tipo transversal realizado com 200 pacientes, maiores de 18 anos, de ambos os sexos, internados nas enfermarias do HC-UFMG e do HRTN e que estavam recebendo atendimento fisioterápico. Ambos são hospitais que oferecem tratamento de patologias de média e alta complexidade. O HC-UFMG 
é referência em transplantes, tratamentos oncológicos, maternidade e berçário de alto risco, marca-passos de alto custo e cirurgias cardíacas. O HRTN atende principalmente demandas nas especialidades clínico-cirúrgicas de urgência e de atendimentos eletivos, além de ser referência no atendimento de pacientes com Acidente Vascular Encefálico (AVE).

Para participar do estudo, os pacientes deveriam ser capazes, ou ter acompanhante capaz, de compreender ou responder perguntas. Os avaliadores perguntavam ao enfermeiro ou fisioterapeuta responsável se o paciente era capaz de acompanhar um diálogo, manter sua atenção pelo período de uma hora e responder de forma precisa às informações solicitadas ${ }^{11}$. Caso fosse verificado que o paciente não seria capaz de responder ao questionário, os avaliadores realizavam os procedimentos com o acompanhante. Esse estudo foi aprovado pelo Comitê de Ética em Pesquisa da Universidade Federal de Minas Gerais (CAAE: 55602216.3.0000.5149) e os participantes assinaram o Termo de Consentimento Livre e Esclarecido.

\section{Instrumentos e procedimentos}

As informações sociodemográficas e clínicas foram coletadas por meio de uma ficha elaborada pelos pesquisadores contendo informações como idade, sexo, escolaridade, local de residência, motivo da internação, tempo de hospitalização, setor de internação, profissionais da reabilitação envolvidos no caso, número de atendimentos fisioterápicos recebidos, reincidência de internações relacionadas à patologia de base e comorbidades. Essas informações foram coletadas por meio de perguntas ao paciente e/ou ao seu acompanhante. Em casos em que esses não souberam responder, as informações foram coletadas nos prontuários.

Os dados funcionais foram coletados utilizando a versão brasileira do "6-clicks", versão reduzida da Activity Measure for Post Acute Care, que apresenta propriedades de medidas adequadas"11 0 "6-clicks" foi desenvolvido com arcabouço teórico da Classificação Internacional de Funcionalidade Incapacidade e Saúde e tem como objetivo avaliar a limitação de atividade de pacientes hospitalizados ${ }^{12}$. O instrumento é de fácil e rápida aplicação, possuindo três domínios: mobilidade básica, atividade diária e cognição aplicada. Esses domínios são independentes entre si e cada um deles possui apenas seis itens relacionados às dificuldades que o paciente apresenta ou à quantidade de ajuda necessária para realizar tarefas dentro do hospital ${ }^{12}$. Os itens são pontuados de 1 a 4 , graduando a necessidade de ajuda como total, muita, pouca ou nenhuma, e o nível de dificuldade como incapaz, muita, pouca ou nenhuma. O escore total de cada domínio varia de 6 a 24, sendo que uma maior pontuação significa maior funcionalidade ${ }^{12}$.

O "6-clicks" é pontuado pelo profissional após a observação direta da realização da tarefa ou do seu julgamento se o avaliado é capaz de realizar o item baseando-se em sua condição clínica. Ainda, o profissional pode avaliar por meio de entrevista ao paciente, ao acompanhante ou outro profissional de saúde que acompanha o caso ${ }^{12}$. Neste estudo, o "6-clicks" foi aplicado por avaliadores previamente treinados por meio de entrevista ao paciente e, quando necessário, aos respectivos acompanhantes. 


\section{Análise estatística}

Para traçar o perfil dos pacientes, foram utilizadas análises descritivas de medidas de tendência central, dispersão e frequência. $O$ teste de normalidade de dados Kolmogorov-Smirnov mostrou uma distribuição não normal de todos as variáveis e por isso foram utilizados diferentes testes não paramétricos de comparação. Para análise de correlação, foi utilizado o teste de Spearman. 0 nível de significância adotado nas análises foi de $5 \%(p<0,05)$. Os dados foram analisados pelo software Statistical Package for Science (SPSS) versão 19.0.

\section{RESULTADOS}

Foram coletados dados de 200 pacientes, sendo 135 internados no HC-UFMG e 65 no HRTN. Do total de questionários, 151 foram respondidos pelos próprios pacientes e 49 pelos acompanhantes. A média de idade dos pacientes foi de $59 \pm 17,13$ anos, sendo 100 do sexo feminino (50\%) e 100 do sexo masculino (50\%). Os pacientes estavam com uma média de $27 \pm 29,93$ dias de internação e tinham recebido $23 \pm 40,08$ atendimentos fisioterápicos. Mais da metade $(58,5 \%)$ já tinha em seu histórico pelo menos uma internação anterior. Outras características da amostra estão apresentadas na Tabela 1.

Tabela 1. Características sociodemográficas e clínicas dos participantes

\begin{tabular}{ll}
\hline Característica & N (\%) \\
\hline Estado civil & $50(25 \%)$ \\
Solteiro & $101(50,5 \%)$ \\
Casado/União estável & $29(14,5 \%)$ \\
Divorciado/Separado & $20(10 \%)$ \\
Viúvo & \\
\hline Escolaridade & $14(7 \%)$ \\
Não alfabetizado & $98(49 \%)$ \\
Fundamental incompleto & $39(19,5 \%)$ \\
Fundamental completo & $42(21 \%)$ \\
Médio completo & $7(3,5 \%)$ \\
Superior completo & \\
\hline Cidade & $96(48 \%)$ \\
Belo Horizonte & $63(31,5 \%)$ \\
Região Metropolitana & $41(20,5 \%)$ \\
Outras & $53(26,5 \%)$ \\
\hline Setor hospitalar & $42(21 \%)$ \\
Cardiovascular & $20(10 \%)$ \\
Neurologia &
\end{tabular}




\begin{tabular}{cl} 
Respiratória & $16(8 \%)$ \\
Oncologia & $30(15 \%)$ \\
Outros & $39(19,5 \%)$ \\
\hline Comorbidades & \\
Ausente & $75(37,5 \%)$ \\
Uma ou duas & $112(56 \%)$ \\
Três ou mais & $13(6,5 \%)$ \\
\hline Profissionais envolvidos & $127(63,5 \%)$ \\
Nutricionista & $66(33 \%)$ \\
Fonoaudiólogo & $45(22,5 \%)$ \\
Terapeuta Ocupacional & $41(20,5 \%)$ \\
Psicólogo & $38(19 \%)$ \\
Assistente social &
\end{tabular}

Notas: Dados apresentados em frequência e porcentagem.

Pouco mais da metade dos pacientes (56\%) era composta por idosos ( $\geq 60$ anos). Dentre esses, pouco mais da metade (57\%) tinha entre 60 a 70 anos, o que equivale a $32 \%$ do total de participantes do estudo. Em relação a ocupação, 39\% eram aposentados, seguidos de 12,5\% domésticas ou diaristas e 9\% profissionais da construção civil. Apenas 3\% da amostra estava desempregada. A renda média relatada foi de aproximadamente um salário mínimo.

Um dos motivos de internação mais recorrentes, com diagnóstico médico confirmado em prontuário, foi o AVE $(11,5 \%)$. Foram encontradas diferentes comorbidades, como Hipertensão Arterial, Diabetes Mellitus, cardiopatias, distúrbios da glândula tireoide, entre outros. Dentre esses, a comorbidade mais encontrada foi a Hipertensão Arterial (45\%) seguida de Diabetes Mellitus (26,5\%).

A Tabela 2 mostra dados relativos à funcionalidade, representada pela mediana por faixa etária dos escores obtidos nos domínios do "6-clicks". Não foram evidenciadas diferenças significativas entre as faixas etárias no domínio de mobilidade básica. Os indivíduos idosos tinham menor funcionalidade no domínio de atividade diária enquanto os mais jovens apresentaram melhores escores no domínio de cognição aplicada. De forma geral, os piores escores foram encontrados no domínio de mobilidade básica, seguido de atividade diária e cognição aplicada. No domínio de mobilidade básica, andar no quarto do hospital e subir escadas foram as atividades que os pacientes apresentaram mais dificuldade. Já no domínio de atividade diária, as tarefas que os indivíduos relataram precisar de mais ajuda foi colocar e tirar roupas da parte inferior do corpo e tomar banho. No domínio de cognição aplicada, a dificuldade mais frequente estava no item cuidar de tarefas complicadas, como administrar uma conta bancária ou providenciar o conserto de equipamentos. 
Tabela 2. Escores obtidos no "6-clicks" por domínio e faixa etária

\begin{tabular}{llll}
\hline Domínio & Faixa etária & Mediana (mínimo-máximo) & Valor $\mathbf{p}$ \\
\hline Mobilidade & 18 a 44 anos & $17(6-24)$ & \\
básica & 45 a 49 anos & $17(6-24)$ & 0,083 \\
& 60 anos ou mais & $14(6-24)$ & \\
\hline \multirow{2}{*}{$\begin{array}{l}\text { Atividade } \\
\text { diária }\end{array}$} & 45 a 44 anos & $21(6-24)$ & $0,034^{\mathrm{a}}$ \\
& 60 anos ou mais & $17,50(6-24)$ & \\
\hline & 18 a 44 anos & $22(6-24)$ & $0,002^{\mathrm{b}}$ \\
$\begin{array}{l}\text { Cognição } \\
\text { aplicada }\end{array}$ & 45 a 49 anos & $20(6-24)$ & \\
\hline
\end{tabular}

Notas: Dados apresentados em mediana, mínimos e máximos; teste não paramétrico de comparação Kruskal Wallis; a=Os indivíduos com 60 anos ou mais obtiveram menores pontuações nesse domínio, as demais faixas etárias foram estatisticamente iguais; b=0s indivíduos de 18 a 44 anos obtiveram maiores resultados nesse domínio, as demais faixas etárias são estatisticamente iguais.

Ao comparar os escores dos três domínios do "6-clicks" - mobilidade básica, atividade diária e cognição aplicada - entre homens e mulheres, não foram encontradas diferenças significativas $(p=0,296 ; p=0,854 ; p=0,548$, respectivamente). Os indivíduos com menor nível de escolaridade apresentaram resultados piores do que aqueles que possuíam ensino médio completo ou mais anos de estudo no domínio de cognição aplicada ( $p=0,027)$.

Os pacientes internados no setor de neurologia apresentaram diferença significativa nos domínios do "6-clicks" e piores escores no domínio de mobilidade básica, seguido de atividade diária e cognição aplicada $(p<0,001)$. Nas demais especialidades, com exceção do setor respiratório que não apresentou diferença entre os domínios $(p=0,782)$, os escores também foram piores no domínio de mobilidade básica. Os domínios de atividade diária e de cognição aplicada não apresentaram diferenças $(p<0,05)$.

Não foi encontrada diferença entre os domínios quando se comparou pacientes com e sem comorbidades $(p>0,100)$ e nem quando se comparou indivíduos reinternados ou não $(p>0,100)$. Os pacientes internados há pelo menos 30 dias apresentaram os piores resultados no domínio de atividade diária $(p<0,05)$. Não houve correlação entre tempo de internação e idade ( $r=-0,106 ; p=0,134)$, bem como entre tempo de internação e sexo $(r=-0,119 ; p=0,092)$.

\section{DISCUSSÃO}

O objetivo deste estudo foi descrever o perfil dos pacientes internados nas enfermarias de dois hospitais 
universitários do município de Belo Horizonte que se encontravam em atendimento fisioterápico. O perfil geral contemplou de forma equilibrada homens e mulheres, em sua maioria de meia idade, casados e com baixa renda e escolaridade. Os hospitais recebem pessoas não só do próprio município, mas da região metropolitana e de outras cidades do estado. Essas características são semelhantes àquelas encontradas em um estudo recente com hospitais públicos mineiros ${ }^{13}$. Em relação à funcionalidade, tarefas de mobilidade básica foram as mais comprometidas, independentemente do setor de internação. Os idosos apresentaram baixos escores em tarefas de atividade diárias e, os indivíduos com menor tempo de escolaridade, em tarefas de cognição aplicada.

Este estudo incluiu a mesma proporção de homens e mulheres, diferentemente das estatísticas nacionais que indicam que mulheres são mais hospitalizadas ${ }^{14}$. Apesar do número total de internações entre as mulheres ser mais alto, ao observar esses dados por faixa etária, mulheres adultas são mais internadas que as idosas e, de forma inversa, homens idosos são mais internados que os adultos. O mesmo pode ter ocorrido com nossos dados, entretanto essa ocorrência não foi analisada. Além disso, os indivíduos incluídos em nossa amostra eram em sua maioria casados. A literatura aponta que indivíduos casados buscam mais assistência médica e atribui uma maior mortalidade aos solteiros ${ }^{15}$, demonstrando que os nossos dados foram condizentes.

Os participantes apresentavam, em sua maioria, baixa escolaridade assim como em outros hospitais públicos ${ }^{13}$ e aqueles com menor tempo de escolarização obtiveram pior escore no domínio de cognição aplicada do "6-clicks", que inclui atividades como acompanhar conversas, lembrar-se onde colocou objetos e gerenciar tarefas complicadas tais como conta bancária. Indivíduos que possuem menor tempo de escolarização realizam menos exames preventivos ${ }^{16}$ e são mais susceptíveis a doenças cardiovasculares ${ }^{17}$. Por outro lado, aqueles com maior tempo de escolarização conseguem tomar melhores decisões relacionadas à saúde, adotando hábitos de vida mais saudáveis ${ }^{18,19}$. A baixa escolaridade pode influenciar a aquisição de patologias e, também, a sua identificação, aumentando a sua gravidade e consequentemente ocasionando maiores taxas de hospitalizações nessa população.

Os participantes internados em quase todos os setores dos hospitais apresentaram maior comprometimento no domínio de mobilidade básica do "6-clicks" que inclui transferências no leito, sentar e levantar de uma cadeira, deambulação no quarto do hospital e subir e descer uma pequena escada. Esses resultados corroboram com a literatura que demonstra uma baixa mobilidade dos pacientes durante a internação $0^{6,20,21,22}$. As lesões que levaram à internação podem repercutir em deficiências de estrutura e função e em limitações em atividade ${ }^{20}$. Outro fator que contribui para essas limitações funcionais é a dor, presente em grande maioria dos pacientes hospitalizados ${ }^{23}$. Como consequência dessas limitações, os pacientes podem apresentar uma maior dependência de cuidadores para realizar suas tarefas funcionais ${ }^{20}$. Portanto, o manejo da dor é fundamental para melhorar a mobilidade dos pacientes hospitalizados, bem como orientações aos cuidadores e a implementação de programas que promovam a independência dos pacientes 
durante a hospitalização.

Pouco mais da metade dos indivíduos internados eram idosos com baixos escores no domínio de atividade diária do "6-clicks", que engloba tarefas como alimentação, vestuário e banho. Tiensoli et al. (2014) também encontraram essa faixa etária mais presente no HC-UFMG. Os idosos estão sujeitos a perdas na sua capacidade de reserva durante a hospitalização, tornando-os mais vulneráveis para apresentarem limitações na sua funcionalidade ${ }^{25}$. Nossos resultados endossam a necessidade de preparar equipes de reabilitação para atender essa população, principalmente proporcionando mais independência nas atividades de vida diária.

Os pacientes estavam internados há quase 30 dias, semelhante à média de outros estudos brasileiros ${ }^{24,26}$. $\mathrm{A}$ literatura aponta uma relação do tempo prolongado de internação com idade mais elevada, já que existe maior incidência de comorbidades em faixas etárias avançadas, retardando a recuperação desses indivíduos ${ }^{26}$. 0 tempo prolongado de internação gera maiores custos para o hospital e ainda está associado à maior incidência de complicações clínicas, mortalidade e incapacidades ${ }^{25,26}$. Em nosso estudo, indivíduos internados há mais de 30 dias obtiveram piores escores no domínio de atividade diária, mas não encontramos correlação entre o tempo de internação e a idade. A ausência de correlação pode ser explicada pela inclusão de diferentes setores dos hospitais, englobando pacientes oncológicos que passam por longos períodos de internação independentemente da idade. Os pacientes internados por tempo prolongado precisam de cuidado especial no que se refere à execução de atividades diárias e de ações para redução do tempo de hospitalização prevenindo assim complicações e diminuindo custos em saúde.

A taxa de reinternação aqui apresentada foi superior a $50 \%$. As readmissões que são potencialmente evitáveis poderiam deixar de ocorrer com um gerenciamento efetivo do quadro clínico e planejamento individualizado da alta com adequação de orientações e recursos para o domicílio ${ }^{25}$. Autores apontam a importância do cuidado pós alta nos 30 primeiros dias para evitar complicações e uma nova internação ${ }^{4}$, além de demonstrarem que os desfechos funcionais na alta predizem reinternações ${ }^{4}$. A proporção de readmissão hospitalar até 30 dias é um indicador usado pelo Ministério da Saúde como parâmetro de qualidade assistencial, o que também evidencia a importância do registro desse dado ${ }^{27}$. Apesar das datas das internações prévias dos pacientes não serem especificadas e da ausência de diferença na funcionalidade em relação a quantidade de internações, a taxa elevada de reinternações serve de alerta aos profissionais para melhorar a função durante a hospitalização e orientar adequadamente o paciente na alta.

As condições neurológicas e cardíacas foram predominantes, endossando as áreas de referência dos hospitais e revelando maior demanda por atendimento fisioterápico nesses setores. Ao analisar as comorbidades dos pacientes, mais da metade possuía uma ou duas doenças além do motivo da internação. As comorbidades mais encontradas foram o diabetes e HAS, que podem estar associadas ao diagnóstico de hospitalização mais encontrado, o AVE. Quanto aos domínios do "6-clicks", verificamos que os escores dos indivíduos com pelo menos uma comorbidade foram iguais 
aos escores daqueles sem comorbidades. Esse resultado indica que a presença de comorbidades não interfere na funcionalidade, mas é importante ressaltar que essas estão associadas ao surgimento de doenças cardiovasculares e mortalidade ${ }^{28} \mathrm{e}$, por isso, requerem atenção dos profissionais de saúde.

Dentre os profissionais da equipe multidisciplinar que também atendiam os pacientes, o nutricionista foi o mais solicitado, seguido do fonoaudiólogo. Essa informação aponta as especialidades de maior demanda e pode orientar a alocação dos recursos humanos do hospital e alertar a equipe quanto ao trabalho interdisciplinar a ser valorizado e desenvolvido. A literatura mostra que o trabalho em equipe dentro de hospitais melhora a comunicação e os desfechos clínicos, limita efeitos adversos, produz maior satisfação ao paciente e diminui o tempo de hospitalização ${ }^{29}$. Os fisioterapeutas como membros dessa equipe devem dialogar com os demais profissionais e atuarem em conjunto para melhor prestação do cuidado aos pacientes hospitalizados.

O tipo de desenho do estudo limita a extrapolação dos nossos resultados e conclusões uma vez que não é possível inferir causalidade. Estudos com acompanhamento longitudinais são importantes e recomendamos sua execução, avaliando os pacientes na admissão, durante a internação e na alta. Isso permitirá observar o impacto da hospitalização, até mesmo em dias. Todos os pacientes selecionados eram atendidos pela fisioterapia, o que pode ter aumentado o número de indivíduos com mais limitações funcionais na amostra. Além disso, não consideramos as faixas etárias para a análise do sexo de nossa amostra. Futuros estudos podem incluir pacientes que não estão em acompanhamento da fisioterapia e também os motivos das limitações na mobilidade desses pacientes para intervenção de reabilitação.

\section{CONSIDERAÇÕES FINAIS}

Os indivíduos internados nos hospitais universitários de grande porte são de meia idade, casada, baixa renda e escolaridade, residentes na região metropolitana de Belo Horizonte. Eles passam por longos períodos de internação, além de apresentarem alta taxa de reinternação e comorbidades. A maior demanda é nos setores de neurologia e cardiovascular, sendo a limitação funcional mais importante em tarefas de mobilidade básica. Os dados encontrados podem nortear o gerenciamento dos recursos humanos e financeiros dos serviços hospitalares públicos, além de contribuir com informações para criar políticas públicas e programas de prevenção e promoção em saúde dentro do hospital. Dessa forma, é possível reduzir as consequências negativas decorrentes da hospitalização e potencializar 0 cuidado dos indivíduos atendidos pelo SUS. 


\section{AGRADECIMENTOS}

Agradecemos a participação e orientação da Profa. Dra. Sheyla Rossana Cavalcanti Furtado, o suporte dos fisioterapeutas do HC-UFMG e do HRTN e o apoio financeiro da Coordenação de Aperfeiçoamento de Pessoal de Nível Superior (CAPES) e da Fundação de Amparo à Pesquisa do Estado de Minas Gerais (FAPEMIG).

\section{REFERÊNCIAS}

1. Goldwater DS, Dharmarajan K, McEwan BS, Krumholz HM. Is Posthospital Syndrome a Result of Hospitalization-Induced Allostatic Overload?. J Hosp Med. 2018;13(5):10.12788/jhm.2986. http://doi:10.12788/jhm.2986

2. Müller M, Grill E, Stier-Jarmer M, Strobl R, Gutenbrunner C, Fialka-Moser V, et al. Validation of the comprehensive ICF Core Sets for patients receiving rehabilitation interventions in the acute care setting. J Rehabil Med. 2011;43(2):92-101. http://10.2340/16501977-0622

3. Lim SER, Dodds R, Bacon D, Sayer AA, Roberts HC. Physical activity among hospitalised older people: insights from upper and lower limb accelerometry. Aging Clin Exp Res. 2018;30(11):1363-1369. http://10.1007/s40520-0180930-0

4. Fisher SR, Graham JE, Krishnan S, Ottenbacher KJ. Predictors of 30-Day Readmission Following Inpatient Rehabilitation for Patients at High Risk for Hospital Readmission. Phys Ther. 2016;96(1):62-70. http://10.2522/ ptj.20150034

5. Smart DA, Dermody G, Coronado ME, Wilson M. Mobility Programs for the Hospitalized Older Adult: A Scoping Review. Gerontol Geriatr Med. 2018;4:2333721418808146. http://10.1177/2333721418808146

6. Mudge AM, McRae P, McHugh K, Griffin L, Hitchen A, Walker J, Cruickshank M, et al. Poor mobility in hospitalized adults of all ages. J Hosp Med. 2016;11(4):289-291. http://10.1002/jhm.2536

7. Zisberg A, Shadmi E, Gur-Yaish N, Tonkikh O, Sinoff G. Hospital-associated functional decline: the role of hospitalization processes beyond individual risk factors. J Am Geriatr Soc. 2015;63(1):55-62. http://10.1111/jgs.13193 
8. Kramer AM, Holthaus D. Uniform patient assessment for post-acute care. Available at: http://cms.gov/medicare/quality-initiatives-patient-assessment-instruments/qualityinitiativesgeninfo/downloads/qualitypacfullreport.pdf. Acesso em: 17 mar. 2016.

9. de Oliveira AP, Mestieri MC, Pontin JC. Epidemiological profile of patients with proximal humerus fracture treated at hospital São Paulo, Brazil. Acta Ortop Bras. 2015;23(5):271-274. http://10.1590/1413-785220152305143730

10. Park S, Thøgersen-Ntoumani C, Ntoumanis N, Stenling A, Fenton SA, Veldhuijzen Van Zanten JJ. Profiles of physical function, physical activity, and sedentary behavior and their associations with mental health in residents of assisted living facilities. Appl Psychol Health Well Being. 2017;9(1):60-80. http://10.1111/aphw.12085.

11. Borges PRT, Sampaio RF, Kirkwood RN, Souza MAP, Mancini MC, Furtado SRC. Reduced version of the Activity Measure for Post-Acute Care (AM-PAC) for inpatients, "6-clicks": Brazilian-Portuguese cross-cultural adaptation and measurement properties. Braz J Phys Ther. 2019;S1413-3555(18)30422-2. http://10.1016/j.bjpt.2019.02.008

12. Jette A, Haley SM, Coster W, Ni PS. Boston University; 2015. AM-PAC Short Forms for Inpatient and Outpatient Settings: Instruction Manual.

13. Gomes LL, Volpe FM. O perfil das internações clínicas e cirúrgicas dos hospitais gerais da rede FHEMIG. Rev Med Minas Gerais 2018;28 (Supl 5): e-S280513

14. Ministério da Saúde (BR). Datasus. Sistema de Informações Hospitalares - Morbidade Hospitalar do SUS [Internet]. Disponível em: http://tabnet.datasus.gov.br/cgi/tabcgi.exe?sih/cnv/nruf.def. Acesso em: 16 set. 2020.

15. Gomes MMF, Turra CM, Figoli MGB, Duarte YAO, Lebrao ML. Asssociação entre mortalidade e estado marital: uma análise para idosos residentes no Município de São Paulo, Brasil, Estudo SABE, 2000 e 2006. 2013; 29(3):56678. http://10.1590/S0102-311X2013000300014

16. Lerhmann-Lerche CS, Larsen SB, Andersen I, Thygesen LC, Kaae Andersen K, Duun-Henriksen AK, et al. Educational level and first-time PSA testing in general practice. Scand J Urol. 2019;53(5):275-281. http://10.1080/21681 805.2019.1681503 
17. Kubota Y, Heiss G, MacLehose RF, Roetker NS, Folsom AR. Association of Educational Attainment With Lifetime Risk of Cardiovascular Disease: The Atherosclerosis Risk in Communities Study. JAMA Intern Med. 2017;177(8):11651172. http://doi:10.1001/jamainternmed.2017.1877

18. World Health Organization (WHO). Health 2020: education and health through the life-course. http://www. euro.who.int/_data/assets/pdf_file/0007/324619/Health-2020-Education-and-health-through-the-life-course-en.pdf?ua=1. Acesso em: 16 set. 2020.

19. Woudstra AJ, Smets EMA, Verdam MGE, Fransen MP. The Role of Health Literacy in Explaining the Relation between Educational Level and Decision Making about Colorectal Cancer Screening. Int J Environ Res Public Health. 2019;16(23):4644. Published 2019 Nov 22. http://10.3390/ijerph16234644

20. Raggi A, Leonardi M, Covelli V, Sattin D, Scaratti C, Schiavolin S, et al. The ICF as a framework to collect and interpret data on the extent and variety of disability in neurological conditions. NeuroRehabilitation. 2015;36(1):17-22. http://10.3233/NRE-141186

21. Silva FM, Pinheiro IM. Avaliação da Cognição, Humor e da Capacidade Funcional em Pacientes Oncogeriátricos Hospitalizados. Revista Kairós: Gerontologia. 2019;22(12):159-174. http://10.23925/2176-901X.2019v22i2p159-174

22. Hartley P, DeWitt AL, Forsyth F, Romero-Ortuno R, Deaton C. Predictors of physical activity in older adults early in an emergency hospital admission: a prospective cohort study. BMC Geriatr. 2020;20(1):177. http://doi:10.1186/ s12877-020-01562-3

23. Münter KH, Clemmesen CG, Foss NB, Palm H, Kristensen MT. Fatigue and pain limit independent mobility and physiotherapy after hip fracture surgery. Disabil Rehabil. 2018;40(15):1808-1816. http://10.1080/09638288.2017.13 14556

24. Tiensoli SD, Bonisson RL, Matozinhos FP, Meléndez GV, Velásquez FSL. Diagnóstico situacional: perfil sociodemográfico e clínico de pacientes internados em unidade de clínica médica. Rev Min Enferm. 2014; 18(3): 573-578. http://10.5935/1415-2762.20140042 
25. Covinsky KE, Pierluissi E, Johnston CB. Hospitalization-associated disability: "She was probably able to ambulate, but l'm not sure". JAMA. 2011;306(16):1782-1793. http://10.1001/jama.2011.1556

26. Rufino GP, Gurgel MG, Pontes TC, Freire E. Avaliação de fatores determinantes do tempo de internação em clínica médica. Revista Brasileira Clínica Médica. 2012;10(4), 291-297.

27. Agência Nacional de Saúde Suplementar. Fichas Técnicas e Nota técnica n 34/DIDES. Rio de Janeiro, 2015. Available at: http://ans.gov.br/images/stories/Plano_de_saude_e_Operadoras/Area_do_prestador/contrato_entre_operadoras_e_prestadores/indicadores-e-nota.pdf. Acesso em: 08 out. 2019.

28. Bozkurt B, Aguilar D, Deswal A, Dunbar SB, Francis GS, Horwich T, et al. Contributory Risk and Management of Comorbidities of Hypertension, Obesity, Diabetes Mellitus, Hyperlipidemia, and Metabolic Syndrome in Chronic Heart Failure: A Scientific Statement From the American Heart Association. Circulation. 2016;134(23):e535-e578. http://10.1161/CIR.0000000000000450

29. Epstein NE. Multidisciplinary in-hospital teams improve patient outcomes: A review. Surg Neurol Int. 2014;5(Suppl 7):S295-S303. http://10.4103/2152-7806.139612 